\title{
Tracking tumor evolution via prostate-specific antigen: an individual post-operative study
}

Mehmet Erbudak ${ }^{1 *}$, Ayşe Erzan²

* Correspondence: erbudak@phys. ethz.ch

'Laboratory for Solid State Physics, ETH Zurich, CH-8093 Zurich,

Switzerland

\begin{abstract}
Background: The progress of the prostate-specific antigen (PSA) level after radical prostatectomy is observed for a patient in order to extract information about the mode of tumor cell growth. Although PSA values are determined routinely to find the doubling time of the prostate marker, to our knowledge, this analysis is the first in the literature.
\end{abstract}

Results: The prostate tumor marker values were determined regularly after the surgery and plotted on a logarithmic scale against time. An initial rapid-growth mode changed to a slower power-law regime within two years of surgery. Our analysis associates this observation with a transition in the growth mode from unrestricted growth of dispersed cells to their clumping into macroscopic structures.

Conclusions: Such studies may help determine the appropriate time window for postoperative therapies in order to increase the life expectancy of the patient.

\section{Background}

Cancer of the prostate gland is one of the most frequently diagnosed male illnesses and may lead to death of the patient. The carcinoma is routinely detected by a straightforward blood test that measures a glycoprotein called prostate-specific antigen (PSA). At an early stage of cancer growth with a localized tumor, radical removal of the prostate gland has proved to be the optimum treatment. If the PSA value rises after radical prostatectomy, different alternatives for treatment are currently under debate. The doubling time (DT) of the PSA value is accepted as a strong prognostic factor for the risk of cancer death. In a group of 379 patients, almost no prostate cancer deaths were recorded within approximately 4 years of prostate removal for $3<$ DT $<8.9$ months, while some patients with DT $<3$ months died within 1.5 years [1]. It is therefore reasonable to infer that findings during the last few years based on long-term statistics suggest a longer life expectancy for patients with postoperative radiotherapy that follows (within 6 months of) radical prostate surgery [2]. Transdermal radiotherapy is commonly applied after the PSA level reaches a threshold value. However, a wait-andwatch method may cost valuable time and the relevant moment for action may be missed regardless of how low the threshold value is set. Many authors have already suggested [3-5] that the entire course of tumor growth offers important information regarding the clinical strategies to be followed.

(c) 2010 Erbudak and Erzan; licensee BioMed Central Ltd. This is an Open Access article distributed under the terms of the Creative Commons Attribution License (http://creativecommons.org/licenses/by/2.0), which permits unrestricted use, distribution, and reproduction in any medium, provided the original work is properly cited. 
The purpose of this work is threefold. (a) To indicate the possibility of detecting fast (exponential) growth of the PSA score well before an arbitrary threshold value is reached, thus gaining time for deciding the therapies to be followed. In principle, this strategy is analogous to determining the DT. (b) To analyze PSA data in a way that reveals a sharp crossover from exponential to power-law growth. (c) To propose a simple model to explain the crossover to slower (power-law) growth.

The second, slower growth regime, we argue, arises because of the coalescence or "condensation" [6] of freely-dividing cancerous cells to form one or more compact tumors, with growth essentially confined to the edges or the surface [4,5,7]. It has been pointed out that at this stage (i) "sensitivity to anti-metabolic drugs decreases, ... (since the fraction of) tumor cells that are in the cell division cycle decreases" [3] and (ii) angiogenesis is expected to start [5].

\section{Results}

\section{Case presentation}

After a radical prostatovesiculectomy (pT2c N0 M0 G2, Gleason $3+4=7$ ) applied to one of us (ME), PSA values were determined with state-of-the-art precision using constant laboratory conditions (Viollier, Brunngasse 6, CH-8401 Winterthur) at time intervals of initially 6 months (see Table 1 ). The error in time measure was \pm 0.5 , while each PSA value was determined with an uncertainty of $\pm 0.002 \mu \mathrm{g} / \mathrm{l}$. In Figure 1 we plot the values listed in the table as a function of time $t$ in months after the surgery. The graph has a characteristic "U" shape, i.e., a shallow increase during the first two years and a steep rise in the last two.

In order to determine the kinetics of cell growth underlying the PSA progression, we present the same PSA values as a function of time in Figure 2a, but plotted on a semi-logarithmic scale. From the time of surgery until about 30 months thereafter we observe a clear linear increase. Analytically, this corresponds to "exponential growth", with the functional form $\sim \exp (p t)$, where $p$ is a constant rate of cell division. This can be determined at an early stage, before an arbitrary threshold value is attained.

Table 1 Measured PSA scores

\begin{tabular}{lll}
\hline Date of the PSA test & Time after the operation (months) & PSA score $(\boldsymbol{\mu g} / \mathbf{l})$ \\
\hline Aug. 2003 & 5 & 0.006 \\
Feb. 2004 & 11 & 0.012 \\
Aug. 2004 & 17 & 0.019 \\
Feb. 2005 & 23 & 0.037 \\
Aug. 2005 & 29 & 0.044 \\
Feb. 2006 & 35 & 0.099 \\
Aug. 2006 & 41 & 0.170 \\
Nov. 2006 & 44 & 0.144 \\
Feb. 2007 & 47 & 0.168 \\
May 2007 & 50 & 0.294 \\
Jul. 2007 & 52 & 0.262 \\
Feb. 2008 & 59 & 0.295 \\
Aug. 2008 & 65 & 0.485 \\
\hline The PSA values and the dates of measurement after the operation in March 2003 as well as the time elapsed thereafter.
\end{tabular}




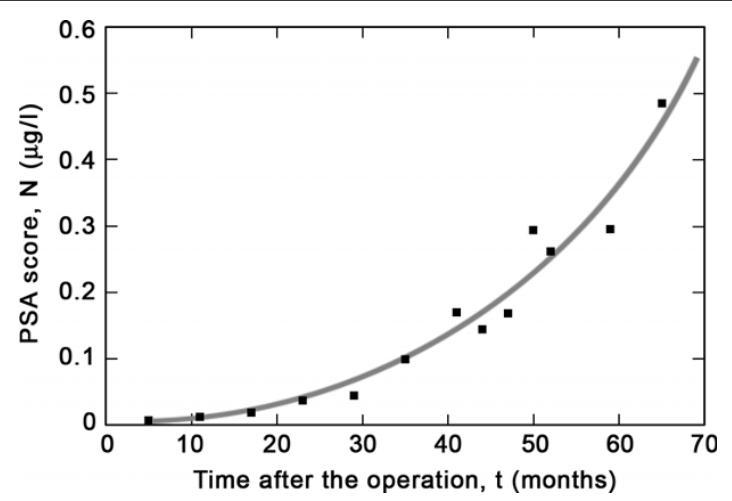

Figure 1 Linear plot of PSA score vs. time. PSA values in $\mu \mathrm{g} / \mathrm{l}$ are displayed as a function of measurement time in months after the surgery.

Later values are noisier and remain below the straight line, implying a different, slower growth law. In Figure $2 \mathrm{~b}$ we examine the departure from exponential behavior by displaying the PSA values vs. time on a log-log plot. Here a straight line signifies a power law (with the functional form $\sim t^{u}$ ). There is sharp crossover from exponential to power-law behavior at about two years after the surgery, rather than a gradual slowing down. From this point on, up to the last measured value, the PSA values grow as a power of the time elapsed after the crossover point.

\section{Growth modes}

We assume that the PSA value is linearly proportional to the number $N$ of carcinoma cells and that, initially, each cell freely divides at a constant rate $p$ (probability per unit time). For such unrestricted growth, the number of cells, $N$, increases by $p N$ per unit time on average, i.e.,

$$
\mathrm{d} N / \mathrm{d} t=p N .
$$

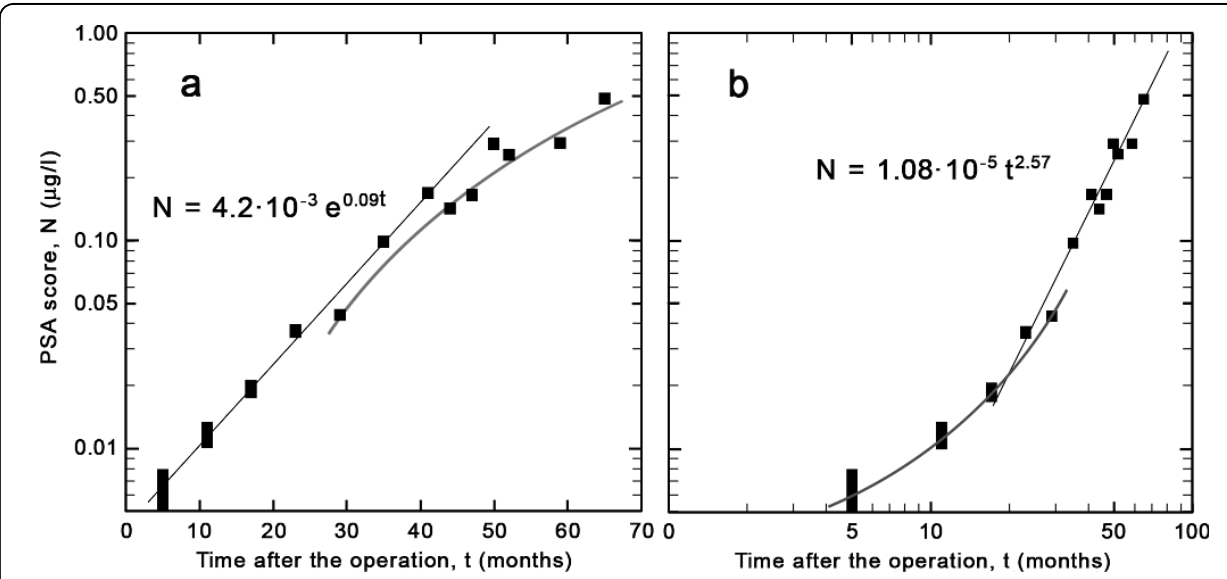

Figure 2 Logarithmic plot of PSA score vs. time. PSA values plotted on a logarithmic scale against (a) linear time and (b) time on a logarithmic scale. Note that the straight line fit in panel (a) signals exponential growth, while that in panel (b) indicates "power-law" growth (see text). The vertical size of the first four points indicates the estimated error, while in the subsequent points the error bars are smaller than the size of the plotted points. 
At any time $t$ from the start of the growth process, $N$ is found to be

$$
N(t)=N_{0} \exp (p t)
$$

where $N_{0}$ is the number at $t=0$. The growth rate $p$ is the only relevant parameter that has to be experimentally determined. In this type of growth, the birth of a new cell has no effect on that of subsequent cells.

As the number of malignant cells grows within the tissue, there must be a spontaneous formation of macroscopic clusters of cells (i.e., tumors) that mop up almost all the microscopic clusters, at a rather sharp transition point, the so-called percolation threshold [8]. We identify this threshold with the crossover observed in Figure $2 \mathrm{~b}$. Once clusters are formed, growth is confined to the surface of the tumor [5] and the kinetic equations should only involve the number $N_{s}$ of actively dividing cells in the surface layer.

The number of cells in the tumor is roughly $N \sim R^{D}$ where $R$ is the average radius and $D$ is the dimension of the cluster. The number in the surface layer will grow as $N_{s}$ $\sim R^{S}$, with $S$ being the surface dimension [9]. In the most general case where $D \leq 3$, Equation 1 is replaced by

$$
\mathrm{d} N / \mathrm{d} t=p k_{1} R^{S}=p k_{2} N^{S / D},
$$

where $k_{1}$ and $k_{2}$ are constant geometrical factors, leading to

$$
N=\left(c_{1}+c_{2} t\right)^{u} .
$$

Here $u=D /(D-S), c_{1}=u N_{0}^{(1 / u)}$, with $N_{o}$ having the same meaning as in Equation 1 , and $c_{2}=p k_{2}$. Irrespective of the exact value of the exponent, for a sufficiently small $c_{1}$, Equation 3 predicts power-law growth. For spheroidal tumors [7] with growth confined to the surface region, $u=3$. The parameter $u$ is related to the compactness or looseness of the clumps of malignant cells and has to be determined experimentally.

\section{Data analysis}

Figure 2a confirms exponential growth of the initial PSA scores with a rate of $p=$ $0.090 \pm 0.004$ per month. Thus, the DT is about 8 months [DT in months is given by $(\ln 2) / \mathrm{p}$ ], or the PSA score increases by more than a factor of three within a year. In current practice, each datum point at a particular time is used by the physician to assess the patient's health condition and to decide upon further action. Although the absolute PSA values are much lower than the widely accepted threshold values for the recurrence of prostate cancer, the growth rate is alarmingly fast. Yet after about three years, the growth rate has slowed down and is seen to deviate from exponential behavior. Nevertheless, any arbitrarily set threshold will eventually be attained.

The log-log plot of the PSA values vs. time in Figure $2 \mathrm{~b}$ shows a crossover from an exponential to a power law. The value of the exponent is $u=2.57 \pm 0.07$, very close to what would be predicted for percolation clusters $[8,9]$ with growth confined to an outer shell.

The values for the coefficient $p$ in the exponential form $\exp (p t)$ and the exponent $u$ in the form $t^{u}$ are found from least squares fits to the linear parts of the plot in the 
respective cases. It should be emphesized that the main import of the paper is not the precise numerical values of $p$ or $u$, although with a linear fit to the straight lines in the two plots these can be determined to an accuracy of two (one) significant digits (digit), respectively, with Pearson's correlation coefficients of $r^{2}=0.98$ and 0.96 . The point is that there is an unambiguous crossover, from a characteristically exponential to a characteristically power-law behavior.

\section{Discussion}

The "Gompertzian" [10] growth curve widely accepted as a "universal law" [3-7,11-14] for the growth of diverse populations including tumors and cell cultures [13] exhibits initial exponential growth, gradually slowing down and finally saturating (in vitro) to a constant value. We find that the data reported in Table 1 are also fitted reasonably well by a Gompertzian (see Figure three of Ref [14]). It would be worthwhile to re-plot the data traditionally analyzed within this gradualist picture on a log-log scale, and see whether the same sharp crossover behavior is actually hiding there as well.

Once the switch to power-law growth occurs, signalling discrete, compact tumors, we can estimate their size assuming that the PSA value is linearly proportional to the total number of malignant cells and knowing the PSA value and tumor size obtained from magnetic-resonance imaging (MRI) at the time of the operation.

In the particular case under study, the gland, prior to its removal, had a diameter of less than $40 \mathrm{~mm}$ (Huber D: MRI Report. Zurich: Klinik Hirslanden; 2002) and the PSA value was $8.5 \mu \mathrm{g} / \mathrm{l}$. The prostate was about $50 \%$ cancerous according to the post-operative biopsy, so we deduce that a PSA level of $0.485 \mu \mathrm{g} / \mathrm{l}$, measured at $t=65$, would correspond to a tumor (consisting only of cancerous cells) approximately $5 \mathrm{~mm}$ in diameter. If not one but two equally-sized compact clusters condense out of the scatter of individual cells, our naive calculation gives a diameter of approximately $4 \mathrm{~mm}$ for each of those tumors. Digital rectal examinations by three independent experts (Brodmann S; Riesterer O; Vollenweider P; 2008) as well as the MRI analysis (Hilfiker P: Medical Report. Zurich: MRI Bethanien; 2008) performed at $t=67$ revealed two masses of about $4 \mathrm{~mm}$ in agreement with our prediction. Subsequently, the subject received 70 Gy of radiation therapy in the anastomosing region during weekdays of $t=68$ and 69 in equal doses, with full bladder and an inflated $(50 \mathrm{ml})$ rectal balloon. The volume was reduced after $46 \mathrm{~Gy}$ in order to spare the vicinity. Since the radiation therapy, the PSA values have remained around $0.16 \mu \mathrm{g} / \mathrm{l}$.

\section{Conclusions}

We find that the PSA values of this patient after surgery follow an initial exponential growth curve, with a sharp crossover to a slower power-law regime at around 20 months. We argue that this may be due to the clumping of individual cancerous cells into macroscopic structures with distinct surfaces, to which subsequent growth is confined.

In the present case, after the onset of the power-law growth regime, it was possible to detect the clusters of cells, i.e., the tumors, via standard imaging techniques, and verify that their sizes coincided with predictions from the model. 
Radiation therapy is not routinely applied after the surgery. The predictive power of our simple analysis, however, makes it highly worthwhile to monitor the PSA scores closely during the so-called wait-and-watch period no matter how low their absolute value is, as has been done in the present case, in order not to miss the optimum time window for post-operative therapy to increase the life expectancy of the cancer patient.

The doubling time is a widely-used characteristic of cell growth and a constant value testifies to exponential growth. The novel aspect of our analysis deals with the deviation from exponential behavior, which transforms into a subsequent power-law regime. Though mathematically convincing, this non-exponential late-stage growth behavior observed for one particular patient may not represent a universal phenomenon. However, monitoring the growth of the PSA value as a function of time may provide the opportunity for observing such a crossover, as for this patient. If such a crossover indeed occurs, it may indicate, as explained above, the formation of macroscopic masses within the tissue, and we suggest that this may be taken as a clue for deciding upon post-operative treatment.

\section{Consent}

Written informed consent for publication was obtained from the patient, who is one of the authors (ME), for publication of this case report and accompanying images. A copy of the written consent is available for review by the Editor-in-Chief of this journal.

\section{Acknowledgements}

ME thanks Drs. M Dubs and P Vollenweider for fruitful discussions during the active surveillance throughout the years before and after prostate surgery. AE would like to thank M Kardar for a helpful comment and also acknowledge partial support by the Turkish Academy of Sciences.

\section{Author details}

${ }^{1}$ Laboratory for Solid State Physics, ETH Zurich, CH-8093 Zurich, Switzerland. ${ }^{2}$ Department of Physics, Faculty of Letters and Sciences, Istanbul Technical University, Maslak, 34469 Istanbul, Turkey.

\section{Authors' contributions}

The authors contributed equally to this work, and read and approved the final manuscript.

\section{Competing interests}

The authors declare that they have no competing interests.

Received: 11 May 2010 Accepted: 30 July 2010 Published: 30 July 2010

\section{References}

1. Freedland SJ, Humphreys EB, Mangold LA, Eisenberger M, Dorey FJ, Walsh PC, Partin AW: Death in patients with recurrent prostate cancer after radical prostatectomy: Prostate-specific antigen doubling time subgroups and their associated contributions to all-cause mortality. J Clin Oncol 2007, 25:1765-71.

2. Bolla M, van Poppel H, Collette L, van Cangh P, Vekemans K, Da Pozzo L, de Reijke TM, Verbaeys A, Bosset J-F, van Velthoven R, Maréchal J-M, Scalliet P, Haustermans K, Piérart M: Postoperative radiotherapy after radical prostatectomy: a randomised controlled trial. Lancet 2005, 366:572-578.

3. Schabel FM Jr: The use of tumor growth kinetics in planning "curative" chemotherapy of advanced solid tumors. Cancer Res 1969, 29:2384-2389.

4. Brú A, Albertos S, Subiza JL, Garcia-Asenjo JL, Brú I: The universal dynamics of tumor growth. Biophys J 2003, 85:2948-2961.

5. Kohandel M, Kardar M, Milosevic M, Sivaloganathan S: Dynamics of tumor growth and combination of antiangiogenic and cytotoxic therapies. Phys Med Biol 2007, 52:3665-3677.

6. Torkington P: Kinetics of deterrence of Gompertzian growth. Bull Math Biol 1983, 45:21-31.

7. Delsanto PP, Guiot C, Degiorgis PG, Condat CA, Mansury Y, Deisboek TS: Growth model for multicellular tumor spheroids. App Phys Lett 2004, 85:4225-4227.

8. Stauffer D, Aharony A: Introduction to Percolation Theory London: Taylor and Francis 1992

9. Mandelbrot BB: The Fractal Geometry of Nature New York: Macmillan 1983.

10. Gompertz B: On the nature of the function expressive of the law of human mortality, and on a new mode of determining the value of life contingencies. Phil Trans $R$ Soc 1825, 115:513-583.

11. DeWys WD: Studies correlating the growth rate of a tumor and its metastases and providing evidence for tumorrelated systemic growth-related factors. Cancer Res 1972, 32:374-379. 
12. Iwata K, Kawasaki K, Shigesada N: A dynamical model for the growth and size distribution of multiple metastatic tumors. J Theor Biol 2000, 203:177-186.

13. Norton L, Simon R, Brereton HD, Bogden AE: Predicting the course of Gompertzian growth. Nature 1976, 264:542-545.

14. Guiot C, Degiorgis PG, Delsanto PP, Gabriele P, Deisboek TS: Does tumor growth follow a "universal law"? J Theor Biol 2005, 225:147-151.

doi:10.1186/1742-4682-7-30

Cite this article as: Erbudak and Erzan: Tracking tumor evolution via prostate-specific antigen: an individual postoperative study. Theoretical Biology and Medical Modelling 2010 7:30.

Submit your next manuscript to BioMed Central and take full advantage of:

- Convenient online submission

- Thorough peer review

- No space constraints or color figure charges

- Immediate publication on acceptance

- Inclusion in PubMed, CAS, Scopus and Google Scholar

- Research which is freely available for redistribution 